\title{
Modeling DNA Opening in the Eukaryotic Transcription Initiation Complexes via Coarse-Grained Models
}

\author{
Genki Shino and Shoji Takada* \\ Department of Biophysics, Graduate School of Science, Kyoto University, Kyoto, Japan
}

Recently, the molecular mechanisms of transcription initiation have been intensively studied. Especially, the cryo-electron microscopy revealed atomic structure details in key states in the eukaryotic transcription initiation. Yet, the dynamic processes of the promoter DNA opening in the pre-initiation complex remain obscured. In this study, based on the three cryo-electron microscopic yeast structures for the closed, open, and initially transcribing complexes, we performed multiscale molecular dynamics (MD) simulations to model structures and dynamic processes of DNA opening. Combining coarse-grained and

OPEN ACCESS

Edited by:

Maciej Maciejczyk,

University of Warmia and Mazury in

Olsztyn, Poland

Reviewed by:

Cezary Czaplewski,

University of Gdansk, Poland Wei Chen, Independent Researcher, Austin, TX, United States

*Correspondence: Shoji Takada

takada@biophys.kyoto-u.ac.jp

Specialty section:

This article was submitted to Biological Modeling and Simulation,

a section of the journal

Frontiers in Molecular Biosciences

Received: 08 September 2021 Accepted: 07 October 2021

Published: 15 November 2021

Citation:

Shino G and Takada S (2021) Modeling

DNA Opening in the Eukaryotic Transcription Initiation Complexes via

Coarse-Grained Models.

Front. Mol. Biosci. 8:772486.

doi: $10.3389 /$ fmolb.2021.772486 all-atom MD simulations, we first obtained the atomic model for the DNA bubble in the open complexes. Then, in the MD simulation from the open to the initially transcribing complexes, we found a previously unidentified intermediate state which is formed by the bottleneck in the fork loop 1 of Pol II: The loop opening triggered the escape from the intermediate, serving as a gatekeeper of the promoter DNA opening. In the initially transcribing complex, the non-template DNA strand passes a groove made of the protrusion, the lobe, and the fork of Rpb2 subunit of Pol II, in which several positively charged and highly conserved residues exhibit key interactions to the non-template DNA strand. The back-mapped all-atom models provided further insights on atomistic interactions such as hydrogen bonding and can be used for future simulations.

Keywords: transcription, eukaryotes, protein-DNA complex, DNA opening, molecular dynamics simulation

\section{INTRODUCTION}

Transcription is fundamental to virtually all area of biology. In eukaryotic cells, RNA polymerase II (Pol II) transcribes all messenger RNAs, making it of central importance. The Pol II transcription initiation requires progressive assembly of several general transcription factors (TFs) and Pol II on the promotor DNA sequence, forming the pre-initiation complex (PIC). After the initial transcription of short RNAs, the transcription machinery escapes the promoter region converting its architecture for the transcription elongation. Much of the transcriptional regulations are related to these early stages of transcription and thus it is of utmost importance to understand the molecular mechanisms of the transcription initiation, which we focus in this study.

Overall processes in the Pol II transcription initiation have been characterized via decades of studies. The PIC consists of Pol II and six general TFs, TFIIA, TFIIB, TFIID, TFIIE, TFIIF, and TFIIH (Buratowski et al., 1989; Roeder, 1996; Grünberg and Hahn, 2013; Sainsbury et al., 2015). In addition, coactivators such as Mediator are involved in its regulation (Schilbach et al., 2017; Nozawa et al., 

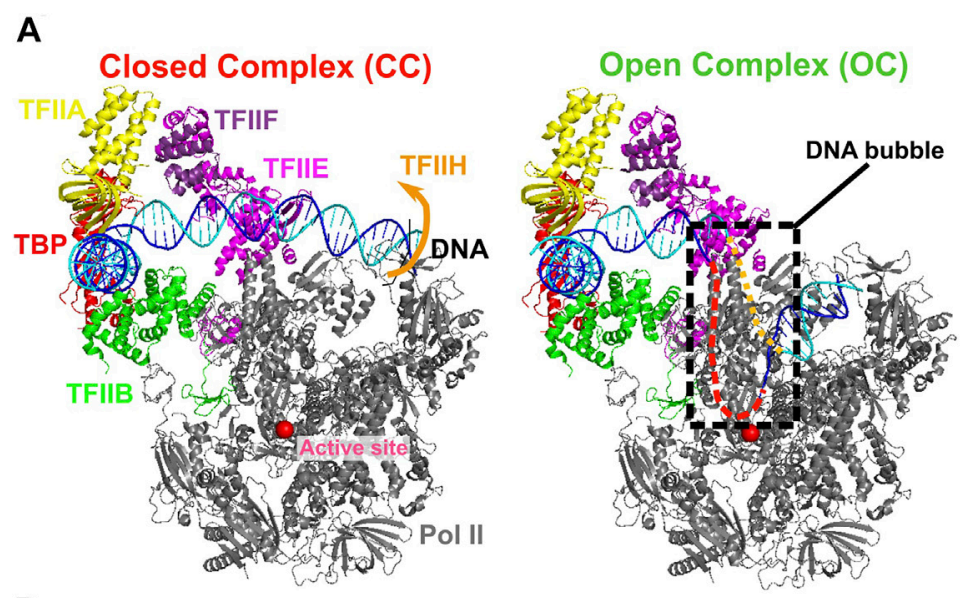

\section{Initially Transcribing Complex (ITC)}

B

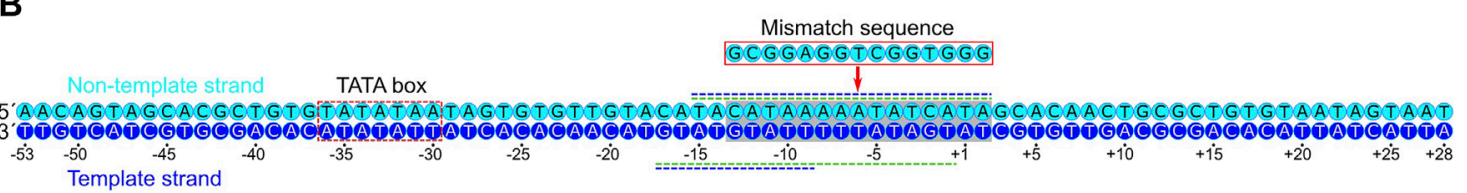

FIGURE 1 | Three yeast PICs of RNA polymerase II and the promoter sequence used. (A) The three PICs obtained by cryo-EM; the closed complex (CC) (PDB: 5FZ5) (left), the open complex (OC) (PDB: 5FYW) (center), and the initially transcribing complex (ITC) (PDB: 4V1N) (right). The CC and OC models contain the promoter DNA, Pol II, TBP, TFIIA, TFIIB, TFIIE, and TFIIF. The ITC model contains the promoter DNA, Pol II, TBP, TFIIB, and 6 bp nascent RNA. Parts of the melted DNA were not modeled in the OC and ITC states (Red and orange broken lines). (B) The promoter DNA sequence used in the current study (numbered relative to the transcription starting site). The sequences are taken from those used in the cryo-EM studies of the OC and the ITC (Plaschka et al., 2016; Plaschka et al., 2015). Blue, the template DNA strand; cyan, the non-template DNA strand; red dashed square, TATA box; gray square, region to which mismatched sequence is introduced in a simulation; green and blue horizontal dashed lines along the sequence, the regions not appeared in the OC and ITC models by cryo-EM, respectively.

2017). The initiation process begins with the recognition of the promoter DNA sequence by TFIID. For the promoter sequences that contain the classic TATA box, the TATA-binding protein (TBP) in TFIID binds the TATA box DNA sequence, leading to 90 -degree bend of the DNA. Then, TFIIA, TFIIB, Pol II-TFIIF complex assemble in this bent site. Further, TFIIE and TFIIH are recruited in order, to form the PIC with the bent duplex DNA (termed the closed complex, CC). In particular, PIC without TFIIH is called as core PIC (cPIC). Next, the promoter DNA melts into the template and non-template DNA strands, driven by the ATP-dependent translocase activity of TFIIH (termed the open complex, OC). The template DNA strand moves toward the active site of Pol II. The melted DNA region is called "DNA bubble", of which size is experimentally characterized as $\sim 6 \mathrm{bp}$ in the OC state (Tomko et al., 2017). Subsequently, the DNA bubble expands to $\sim 13$ bp (Tomko et al., 2017), which allows the template DNA strand reaching to the active site to begin the messenger RNA synthesis. The complex in which the initial transcription begins is called the initially transcribing complex (ITC). Notably, while the ATP-dependent translocase activity of TFIIH facilitates the promoter DNA opening (Compe and Egly, 2012; Fishburn et al., 2015), some promoter DNAs can open spontaneously without TFIIH (Plaschka et al., 2016).

Recently, the cryo-electron microscopy (cryo-EM) revealed near-atomic structures in key stages of the Pol II transcription initiation (Schilbach et al., 2017; Nozawa et al., 2017; Plaschka et al., 2016), which provided the model of DNA opening process in the PICs (Figure 1A) (Plaschka et al., 2016). The model is based on the yeast CC, OC (Plaschka et al., 2016), and ITC structures (Plaschka et al., 2015), and the highly conserved human CC structure (He et al., 2013). However, the state transitions from CC to OC, and to ITC were not directly observed. Moreover, the modeled structures of OC and ITC by cryo-EM do not contain parts of DNA strands because of high flexibility in the DNA bubble. Thus, how the template and nontemplate DNA strands behave inside Pol II has not been fully understood. Complementarily, the DNA bubble size in OC and ITC states has been detected via optical and magnetic tweezer experiments (Tomko et al., 2017; Fazal et al., 2015). However, the structural details in the DNA bubble is currently missing.

Given such situations, molecular dynamics (MD) simulations can offer another complementary approach to address the structural dynamics of the Pol II transcription initiation since MD simulations can provide high-resolution spatiotemporal information (Chen et al., 2010; Hyeon and Thirumalai, 2011; Feig and Burton, 2010; Huang et al., 2010; Silva et al., 2014). However, since the DNA opening process involves rather largescale and slow movements of DNA within large complexes, conventional $\mathrm{MD}$ simulations with fully-atomic resolution (designated as the all-atom (AA) MD hereafter) cannot easily sample these structural dynamics. To circumvent this difficulty, one can alternatively use coarse-grained (CG) MD simulations, which can speed up the simulation by orders of magnitude at the cost of accuracy (Liwo et al., 2014; Takada et al., 2015; Kmiecik et al., 2016; Pak and Voth, 2018). Once comprehensively sampled by CG-MD, one can back-map the sampled CG structure models 
into AA models, followed by AA-MD simulations (Shimizu and Takada, 2018). A recent study employed such a protocol to gain comprehensive and high-resolution energy landscape in a bacterial RNA polymerase (Unarta et al., 2021).

In this study, using the cryo-EM yeast structures for the CC, OC, and ITC, we performed multiscale MD simulations to model structures and dynamic processes of DNA opening.

Combining CG- and AA- MD simulations, we first obtained the atomic model for the DNA bubble in the OC. Then, in the CG-MD simulation from the OC to the ITC, we found a previously unidentified intermediate state which is formed by the bottleneck in the fork loop 1 of Pol II: The loop opening triggered the escape from the intermediate, serving as a gatekeeper of the promoter DNA opening. In the ITC, the non-template DNA strand passes a groove made of the protrusion, the lobe, and the fork of Rpb2 subunit of Pol II, in which several positively charged and highly conserved residues exhibit key interaction to the non-template DNA strand.

\section{MATERIALS AND METHODS}

\section{Preparation of the Simulation System}

We modeled the three yeast structures, CC, OC, and ITC, based on the cryo-EM structure models, 5FZ5 (Plaschka et al., 2016) and 6GYL (Dienemann et al., 2018) for CC, 5FYW (Plaschka et al., 2016) for OC, and $4 \mathrm{~V} 1 \mathrm{~N}$ (Plaschka et al., 2015) for ITC. Missing residues in the original models were modeled by the software MODELLER (Webb and Sali, 2016; Marti-Renom et al., 2000; Sali and Blundell, 1993; Fiser et al., 2000).

We used the DNA sequence identical to that used in cryo-EM studies (Plaschka et al., 2016; Plaschka et al., 2015). The sequence was derived from the promoter sequence of HIS4 gene locus, from which $28 \mathrm{bp}$ were deleted at the downstream of the TATA box.

\section{Coarse-Grained MD Simulations}

In this study, we applied the coarse-grained (CG) simulation model that has been developed previously and extensively applied to protein-DNA complex systems (Levy et al., 2007; Terakawa et al., 2012; Freeman et al., 2014; Zhang et al., 2016; Shimizu et al., 2016; Lequieu et al., 2017; Niina et al., 2017; Brandani et al., 2018; Tan and Takada, 2020). We used AICG2+ model for proteins, 3SPN.2 model for DNA (Li et al., 2014; Hinckley et al., 2013). Briefly, in AICG2+, each amino acid in proteins are represented by one CG particle placed at its $\mathrm{Ca}$ position and the structurebased contact potential biases its energy landscape towards the reference structure. In 3SPN.2 model, each nucleotide is modeled by three CG particles corresponding to the phosphate, the sugar, and the base. Orientation-dependent potentials for base-base interactions and others are designed to reproduce basic experimentally-characterized properties of duplex and, to some extent, single strands. Between proteins and DNA, we applied the structure-based contact potential for representing the specific interactions, as well as a general excluded volume term and the electrostatic interaction via the Debye-Huckel approximation (the monovalent salt concentration was set to $200 \mathrm{mM}$ throughout this study). For the electrostatic interaction, we employed partial charges on the surface residues of proteins, which were optimized to reproduce the electrostatic potential around the protein obtained by the all-atom model via the RESPAC method (Terakawa and Takada, 2014). For time propagation, including the solvent effect implicitly, we employed a simple Langevin dynamics at the temperature $300 \mathrm{~K}$. For all the CG-MD simulations, we used the software CafeMol 3.2 (Kenzaki et al., 2011).

The specific protein-DNA interaction, i.e., the structure-based contact potential is, as usual, expressed as

$$
\sum_{i j \in \text { contact }} \epsilon_{g o}\left[5\left(\frac{\boldsymbol{r}_{i j 0}}{\boldsymbol{r}_{i j}}\right)^{12}-6\left(\frac{\boldsymbol{r}_{i j 0}}{\boldsymbol{r}_{i j}}\right)^{10}\right]
$$

where $r_{i j}$ is the distance between CG particles $i$ and $j, r_{i j 0}$ is the corresponding distance at its reference structure, and $\epsilon_{g o}$ is a parameter that modulates the strength of the interaction, of which value was calibrated to be $1.2 \mathrm{kcal} / \mathrm{mol}$, to maintain experimentally characterized contacts in the three states, CC, OC, and ITC (Plaschka et al., 2016; Plaschka et al., 2015). Specifically, we set the following four conditions to be satisfied:

1. In the CC state, the contact between DNA and the E-wing of TFIIE is maintained.

2. In the OC state, the template DNA strand can maintain the native contacts with a region close to the active site of Pol II.

3. In the OC and ITC states, an upstream side of DNA maintains its contact with N50, K51, and T52 of TFIIE.

4. In the OC and ITC states, the triple mutations N50E, K51E, and T52E lead to loss of the DNA-TFIIE contacts (Plaschka et al., 2016).

For the specific protein-DNA interaction, we collected protein-DNA contacts in the three complexes structures and used its union set for the structure-based contact potential. This union set includes the particular contacts satisfying above four conditions.

\section{Trajectory Analysis}

The state-to-state transitions were characterized by protein-DNA contacts that depend on the state. There are 92, 22, and 118 contacts between proteins and DNA in CC, OC, and ITC states, respectively. The contacts in $\mathrm{CC}$ are all specific to the $\mathrm{CC}$ state and are not shared with the other two states. Thus, all the 92 contacts are used to characterize the CC state. The contacts in OC are mostly a subset of the contacts in the ITC state (19 out of 22 included in the ITC contacts). We used all the 22 contacts to characterize the OC state. Of the 118 contacts in ITC states, 99 are unique to the ITC state and thus are used to characterize the ITC state. Once the sets of contacts are defined, we quantify the stateto-state transition by the fraction of contacts formed in each snapshot.

The size of the DNA bubble was estimated as the sum of the broken base pairs, which are defined by the distance between the CG particles of the base pairs larger than $6.2 \AA$ : In preliminary CG-MD simulations of duplex DNA at the same solvent 
condition, the probability that the base-base distance is larger than $6.2 \AA$ was $0.3 \%$. In apparently melted DNA configurations, their base-base distances were almost surely larger than this threshold distance.

\section{Back-Mapping to All-Atom Model and All-Atom MD Simulations}

Following the previously developed protocol (Shimizu and Takada, 2018), we performed the back-mapping from our CG models to all-atom models. While we used the cryo-EM-based $\mathrm{CC}$ protein structures as the reference structures of all the three states in the dynamical modeling, we moved them back to the respective cryo-EM protein structures aiming at more accurate modeling of all-atom structures. For the intermediate state $\mathrm{I}_{2}$, we used the OC structure as the reference. For each state, we began with the CG-MD simulation at $300 \mathrm{~K}$ for $10^{5} \mathrm{MD}$ steps. Then, to reduce local fluctuations, we performed a quick annealing simulation, quenching the temperature from 300 to $1 \mathrm{~K}$, followed by a $10^{5} \mathrm{MD}$ step simulation at $1 \mathrm{~K}$. The final structure was put into the back-mapping toolset. For DNA, we applied the CG to AA reconstruction tool (Shimizu and Takada, 2018), whereas for proteins, we employed the PD2 ca2main (Moore et al., 2013) for backbone and SCWRL4 (Krivov et al., 2009) for sidechain reconstruction.

Once the all-atom model for the PIC were obtained, we performed all-atom $\mathrm{MD}$ simulations using the software GROMACS 2020.2 (Abraham et al., 2015) with the protein, DNA, and water force fields, ff14SB (Maier et al., 2015), and parmbsc1 (Ivani et al., 2016), and TIP3P (Jorgensen et al., 1983), respectively. We used the standard protocol: We set the box size of $182.2 \times 232.1 \times 186.4 \AA^{3}$ solvating with water molecules and $171 \mathrm{Na}^{+}$ions to neutralize the system. After the energy minimization, we equilibrated the local system with NVT and then NPT ensembles ( $\mathrm{T}=300[\mathrm{~K}]$, the pressure $1 \mathrm{bar}$ ), followed by $10 \mathrm{~ns} \mathrm{MD}$ simulations. We used the cutoff distance of $1 \mathrm{~nm}$ for the Coulomb interaction with the particle-mesh-Ewald for long range treatment.

\section{RESULTS}

\section{Multiscale Modeling of Pre-Initiation Complexes}

Our multiscale modeling begins with CG-MD simulations that connect the three states of the PIC; the CC, OC, and ITC. The constructed CG models were then back-mapped to AA models, which is followed by short-time MD simulations with the AA models.

We employ the CG model that has been extensively used to protein-DNA complexes (Levy et al., 2007; Terakawa et al., 2012; Freeman et al., 2014; Zhang et al., 2016; Shimizu et al., 2016; Lequieu et al., 2017; Niina et al., 2017; Brandani et al., 2018; Tan and Takada, 2020). In the CG model, each amino acid in proteins is represented as one particle located at the $\mathrm{Ca}$ atom position and each nucleotide in DNA is modeled by three particles each representing the phosphate, the sugar, and the base. The protein energy function AICG2+ contains the contact potentials that stabilizes the predefined reference (native) structure, i.e., the structure-based model (Li et al., 2012). The DNA energy function 3SPN.2 is empirically tuned to reproduce several experimental data such as the sequence-dependent melting temperature and bending modulus (Hinckley et al., 2013). The protein-DNA energy function consists of the generic terms; the short-range repulsion and the electrostatic interaction, and the specific interactions; structure-based contact potentials (See Materials and Methods for more details).

The simulation system consists of an 81-bp promoter DNA (the sequence shown in Figure 1B) and the protein complex that contains Pol II, TBP, TFIIA, TFIIB, TFIIE, and TFIIF (that is, this study deals with cPIC). TFIIH is not included because the DNA bubble can form without TFIIH (Plaschka et al., 2016; Alekseev et al., 2017) and because the structural information on ATPdependent conformational change in TFIIH is incomplete albeit some structures previously reported (Schilbach et al., 2017; Dienemann et al., 2018; Osman and Cramer, 2020).

The root-mean-square-differences (RMSD) of protein complexes were $0.85 \AA$ between CC and OC, and $3.5 \AA$ between OC and ITC, which are smaller than the resolutions reported in the cryo-EM analysis $(8.8,4.4$, and $7.8 \AA$, for CC, OC, and ITC models, respectively). We note that we excluded DNA in the calculations of these RMSDs. In the CG-MD simulations, these modest-sized structure changes in proteins should appear via the interaction to DNA (and a short RNA in the case of ITC). Since CC contains the weakest protein-DNA interaction among the three complexes structure models, we took the protein structure of $\mathrm{CC}$ as a reference structure of the protein complex in the CG model throughout this study.

\section{Modeling the DNA Bubble in the Open Complex}

First, to obtain the OC model with the open DNA, we performed 40 independent CG-MD simulations of $5 \times 10^{6} \mathrm{MD}$ steps, starting from the CC structure (Supplementary Figure S1). In any of the simulations, the promotor DNA did not melt spontaneously and most of the OC specific protein-DNA contact did not appear although the particular region of the promotor $(-18 \sim+7$ relative to the transcription start site (TSS)) was distorted toward the cleft of Pol II (Supplementary Figure S1). This distortion in DNA was observed in a previous cryo-EM study, which indicates the prestage of DNA opening (Dienemann et al., 2018). A previous study shows that the DNA opening in the absence of TFIIH takes a very long time; the real-time observation of the formation of the DNA bubble shows that it takes a few seconds (Fazal et al., 2015). Therefore, it is reasonable that we did not observe spontaneous DNA opening in our CG-MD simulations that cannot cover second time scales.

Then, to enforce the prompt DNA opening, we modified the non-template DNA sequence to introduce the DNA mismatch of 15 bases into the promotor (Figure 1B). The introduced mismatch is identical to that used for the cryo-EM structures of OC and ITC (Plaschka et al., 2016; Plaschka et al., 2015). Under this condition, we performed 40-independent CG-MD 

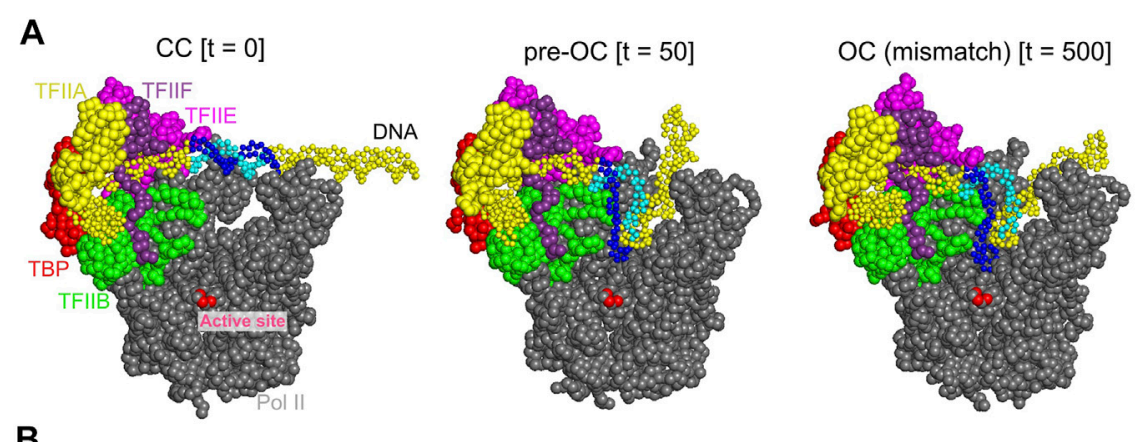

B
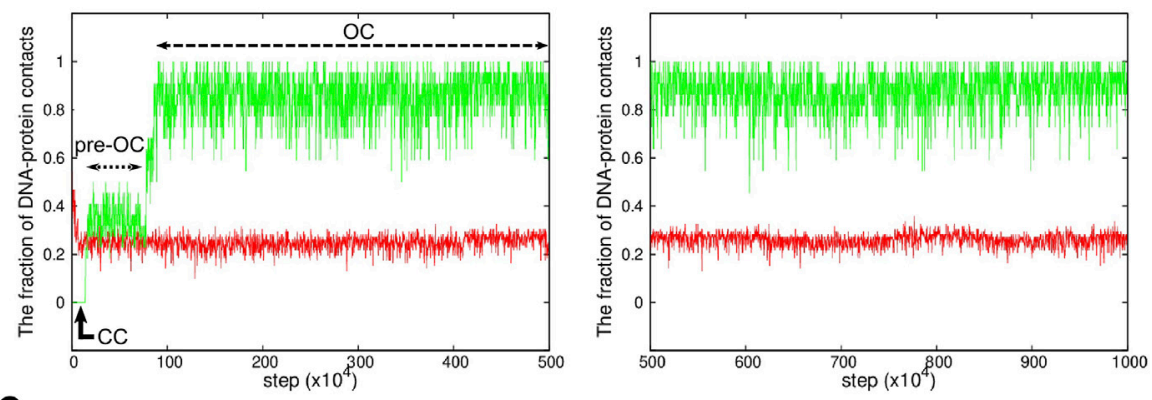

C
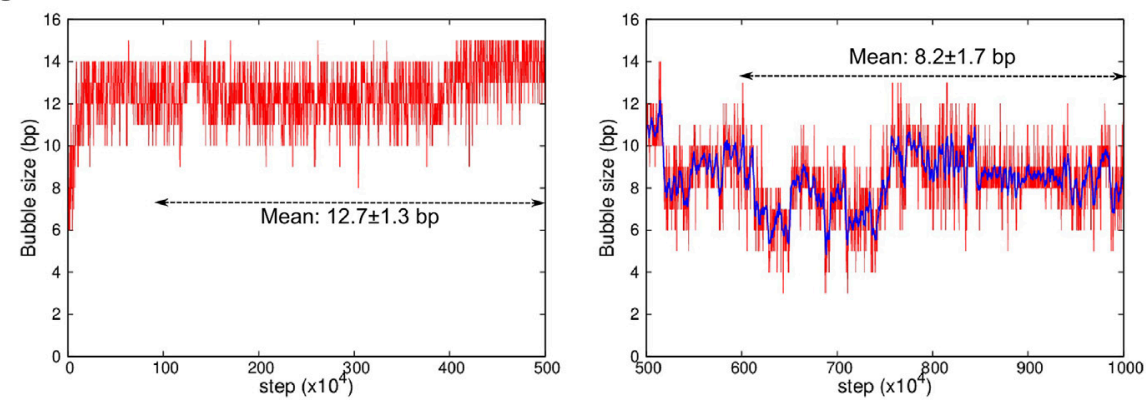

FIGURE 2 |Coarse-grained MD simulation for the transition from the CC to OC states. Results of a representative trajectory are shown. (A) Snapshots at 0 MD step (left, the CC state), at $50 \times 10^{4} \mathrm{MD}$ steps (center, the pre-OC state), and at $500 \times 10^{4} \mathrm{MD}$ steps (right, the OC state with the mismatch). Some proteins are not displayed to make DNA visible. The same colors are used as Figure 1A. Blue and cyan region in DNA indicates the 15-bp mismatch region, forming the DNA bubble. (B) The time course of the fractions of protein-DNA contacts specific to CC (red) and OC (green). (C) The time course of the DNA bubble size. In (B,C), the left/right panels are from the first/second halves of MD simulations with/without the DNA mismatch. The blue curve in the right panel in (C) shows a moving average over 11 points.

simulations of $2 \times 10^{7} \mathrm{MD}$ steps (a representative trajectory is shown in Figure 2). Figure 2B, left panel depicts a representative time course of the fraction of protein-DNA contacts specific to CC (red) and to OC (green). In this trajectory, in the very initial phase, $\sim 80 \%$ of the CC-specific contacts were lost, whereas $\sim 40 \%$ of the OC-specific contacts were formed to reach an intermediate state, which we call "pre-OC" state (Figure 2A, center). In the pre-OC state, most of the mismatched DNA region melted to form a bubble of $\sim 13 \mathrm{bp}$ (Figure $2 \mathrm{C}$ ). This caused the +2 site of the template DNA strand to form new contacts with Pol II (Supplementary Movie S1). All the 40 trajectories paused at this pre-OC state (40/40 cases). In the trajectory in Figure 2B, left panel, the template DNA strand jumped further down to the active site at $\sim 0.9 \times 10^{6} \mathrm{MD}$ steps, reaching to the OC-like state with the mismatch (Figure 2A, right, Figure 2B) (22/40 cases). In the 22 cases, we observed that the complex further moved towards the ITC state in five cases). The transition was driven by the new contact formation of the +1 site of the template DNA strand with Pol II (Supplementary Movie S1).

To obtain the OC structure model without the DNA mismatch, for obtained OC-like structures with the mismatch, we changed the DNA sequence back to the original sequence without mismatch, followed by $5 \times 10^{6} \mathrm{MD}$ steps CG-MD simulations. A representative trajectory is depicted in the right panels of Figures 2B,C. While the overall positioning of DNA did not change (Figure 2B, right), part of the melted DNA regained the base pairing during the trajectory (Figure 2C, right). The observed bubble size fluctuated in time in the range of 6-10 bp, with the mean and the standard deviation $8.2 \pm 1.7 \mathrm{bp}$. In the 22 cases, we did not see significant difference in the fraction of DNAprotein contacts and the DNA bubble size (Another trajectory shown in Supplementary Figure S2). Notably, we observed that 


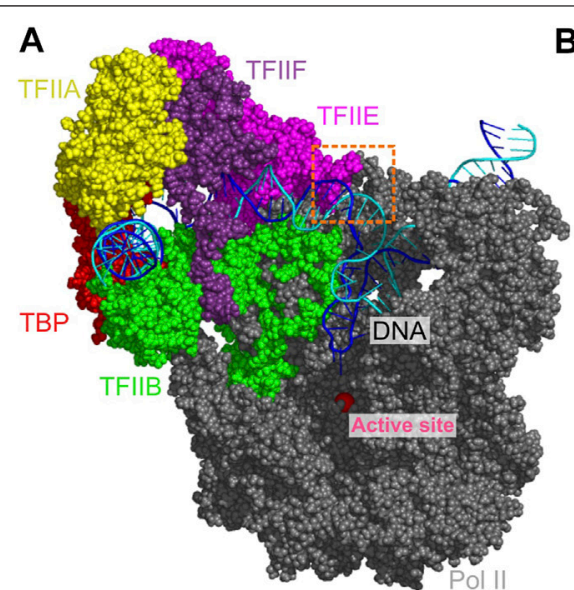

C

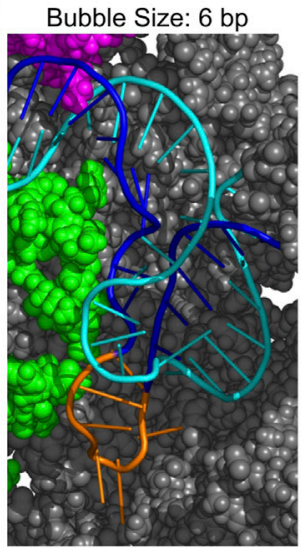

B

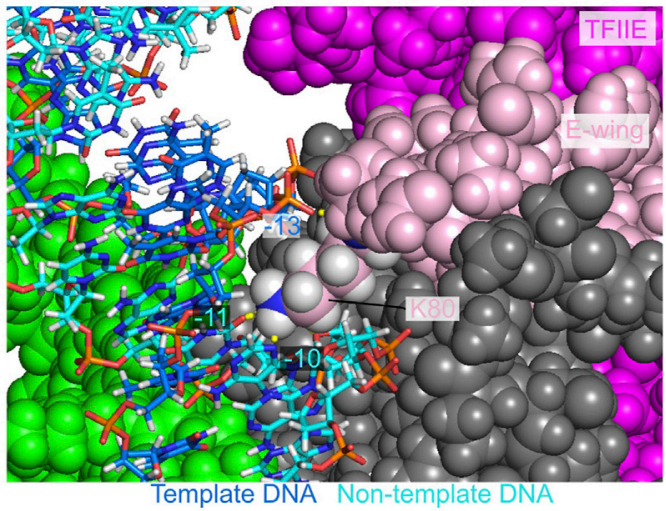

Bubble Size: 9 bp

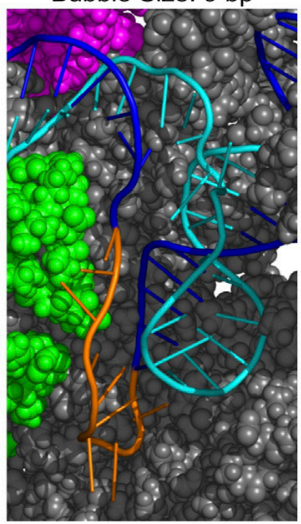

FIGURE 3 | The open DNA in the OC state of PIC. (A) Atomic structure model for the OC state. Some proteins are not displayed to make the DNA visible. (B) A close-up view of the orange dashed squared area in (A). Pink, the E-wing of TFIIE; yellow dashed lines, hydrogen bonds between DNA and the E-wing. (C) Open DNA structures with the bubble size of 6 bp (left) and 9 bp (right). Orange, the template DNA strand in the bubble.

the bubble size depends on the promoter sequence to some extent; with the promoter sequence used in the single-molecule magnetic tweezer experiment, our simulation resulted in the bubble size of $5.5 \pm 1.4 \mathrm{bp}$, which is fairly compared with the experimental estimate, $6.1 \pm 0.3 \mathrm{bp}$ (Tomko et al., 2017).

To detect protein-DNA interactions in the OC state at atomic level, we modeled all-atom structures via back-mapping from the snapshots of a CG-MD trajectory with the DNA bubble sizes of 6 and $9 \mathrm{bp}$. The obtained all-atom models were further relaxed/ refined by $10 \mathrm{~ns} \mathrm{MD}$ simulations with explicit water solvent (Figure 3). In the upstream of the DNA bubble, we find that the hydrogen bonds of the TFIIE E-wing residues K80 with the non-template DNA at -11 to -10 sites, and with the template DNA at -13 site, which are present in the CC state, are maintained (Figure 3B). These interactions are suggested to facilitate the promoter opening and contribute to the efficiency of transcription initiation (Forget et al., 2004). Comparing the structures with 6 and 9 bp DNA bubbles, we find that the template DNA strand is rather similar each other while the non-template DNA strand is more mobile. The $6 \mathrm{bp}$ in the downstream side (from -4 to +2 sites) were melted in both structures, while the $3 \mathrm{bp}$ in the upstream side (from -7 to -5 sites) were formed/melted in the $6 \mathrm{bp} / 9 \mathrm{bp}$ DNA bubbles.

\section{Dynamical Modeling of the Transition from the Open Complex to the Initially Transcribing Complex}

Next, we addressed dynamic process of the transition from the OC state to the ITC state. Starting from a final snapshot of the previous simulation that paused at the OC state for the promoter DNA without the DNA mismatch, we performed 140 independent CG-MD simulations of $2 \times 10^{7} \mathrm{MD}$ steps (Figure 4). In most trajectories (130/140 cases), the contact between the promoter DNA (from -16 to -9 sites) and the E-wing of TFIIE persisted for the whole simulation time, which clearly precluded the template DNA strand from accessing the active site. Only in 10 cases, we observed the disruption of this contact, which directly triggered the template DNA strand to move down towards the active site (Figure 4). 9 out of these 10 trajectories reached the ITC state.

In these successful trajectories, we found two intermediate states (Figure $\mathbf{4 A}$ the second and the third models) before 

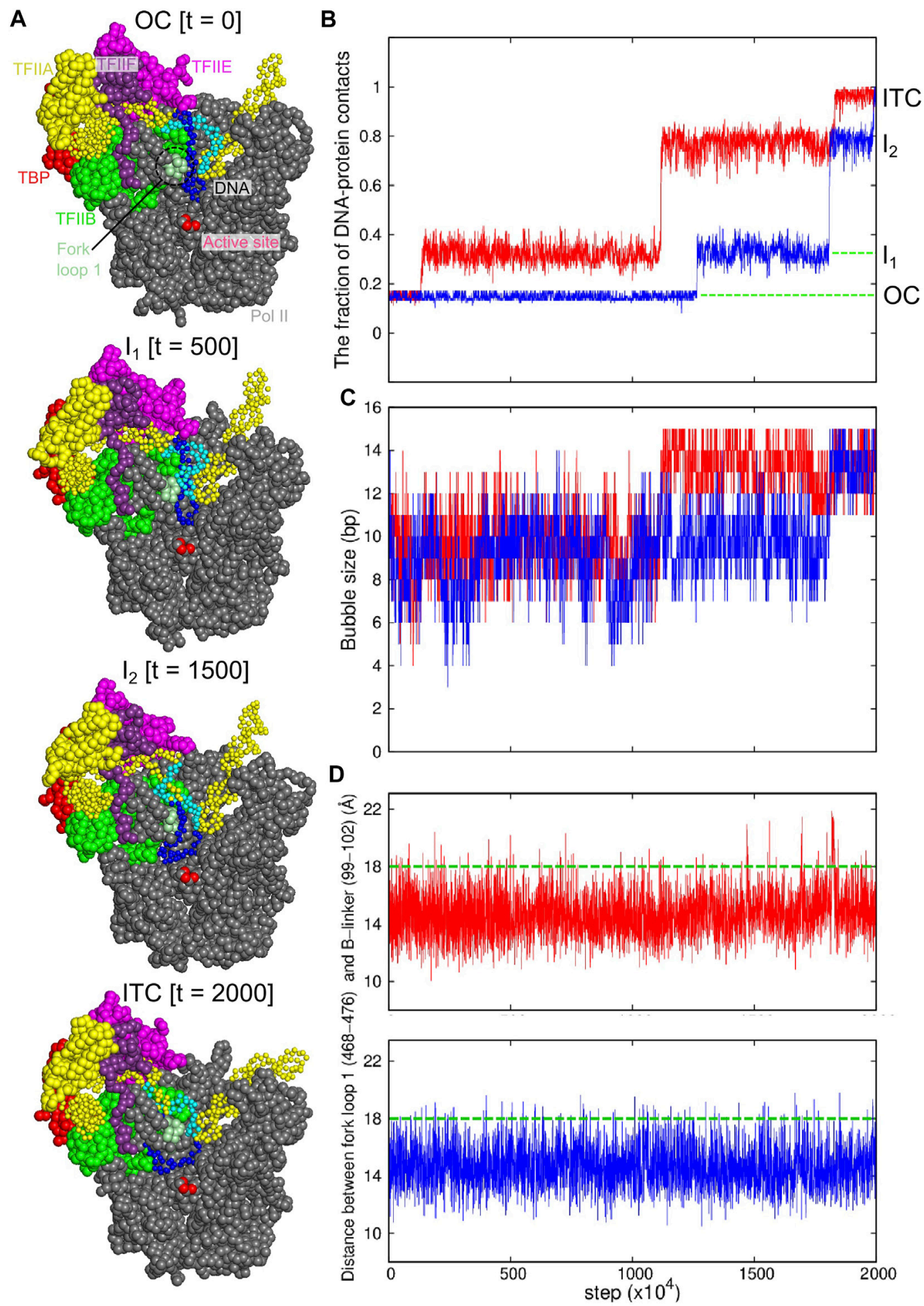

FIGURE 4 | Coarse-grained MD simulation for the transition from the OC to ITC states. Results of two representative trajectories are shown in red and blue curves. (A) Snapshots from the red trajectory at 0 MD step (top, the OC state), at $500 \times 10^{4} \mathrm{MD}$ steps ( $\mathrm{I}_{1}$ state), at $1500 \times 10^{4} \mathrm{MD}$ steps (the $\mathrm{I}_{2}$ state), and $2000 \times 10^{4} \mathrm{MD}$ steps (bottom, the ITC state). (B) The time course of the fractions of protein-DNA contacts specific to ITC. (C) The time course of the DNA bubble size. (D) The time courses of the distance between the centers of mass of the fork loop 1 of the Pol II Rpb2 (468-476 residues) and the B-linker in TFIIB (99-102 residues). Green dashed lines, a characteristic distance for the template DNA to pass through the fork loop 1. 

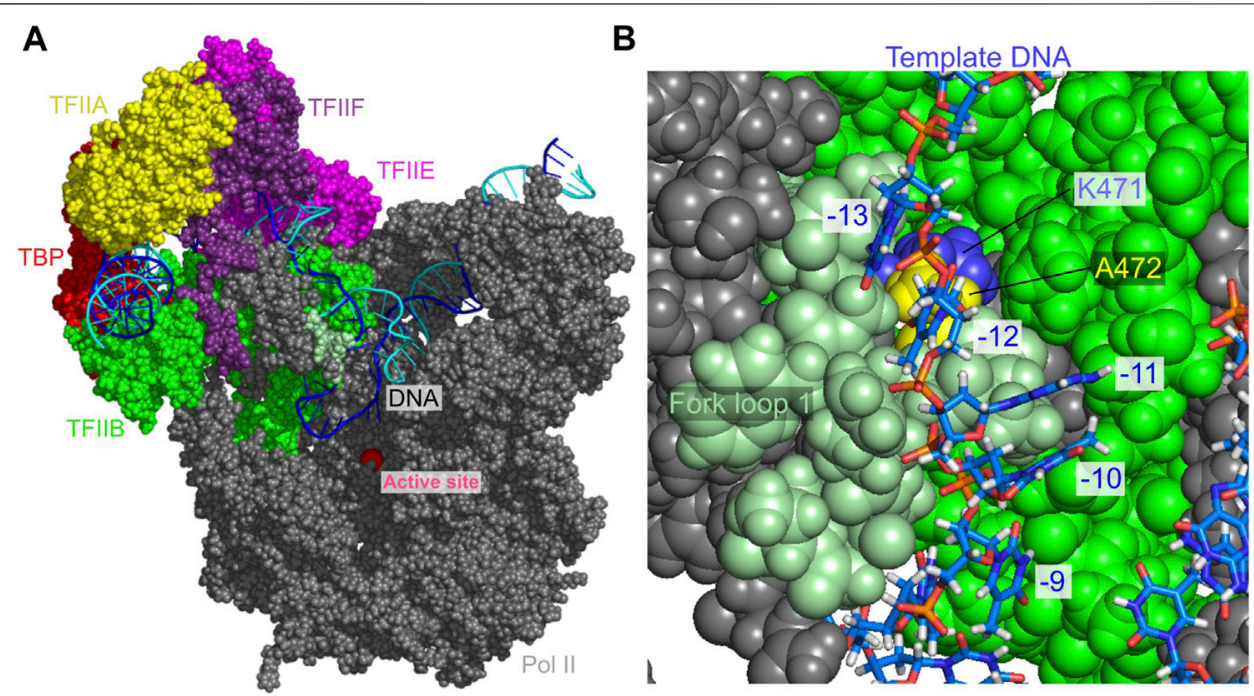

C
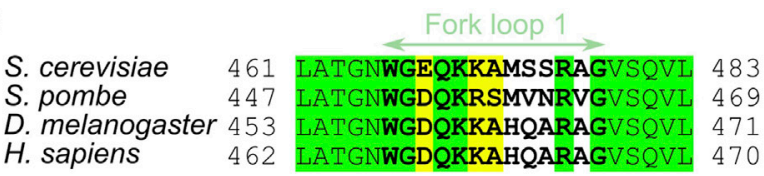

FIGURE 5| The open DNA in the intermediate state $I_{2}$. (A) The atomic structure model for the $\mathrm{I}_{2}$ state. Some proteins are not displayed to make the DNA visible. (B) The close-up view of the fork loop 1 (pale green) that blocks the template DNA passage. (C) Multiple sequence alignment of the fork loop 1 region of the Rpb2. Green, invariant; yellow, conserved.

reaching the ITC state (Figure $\mathbf{4 A}$, bottom). In a representative trajectory (red in Figures 4B-D), the first transition occurred at $\sim 1$ $\times 10^{6}$ MD steps, after which about $35 \%$ of the ITC specific contacts were formed. In this intermediate state $I_{1}, \sim 4$ bp of the template DNA strand, $(-2 \sim+2$ sites, relative to the TSS $)$ approached the active site, while the contact between the DNA and the TFIIE E-wing is maintained (Figure $\mathbf{4 A}$ the second structure). After a long waiting time, the DNA was detached from the TFIIE E-wing region (at $1.1 \times 10^{7} \mathrm{MD}$ steps in the red trajectory), followed by the motion of the entire DNA bubble towards the active site (Figure 4A the third structure). However, the template strand DNA in the upstream side of the DNA bubble, $-13 \sim-9$ sites, collides with the fork loop 1 of Rpb2 subunit of Pol II (Figure 4A, the third structure, and Supplementary Figure S4, left). This forms a metastable intermediate state $\mathrm{I}_{2}$. After some duration time at this intermediate state, the complex made the final transition to the ITC state (at $\sim 1.7 \times 10^{7} \mathrm{MD}$ steps in the red trajectory). The other successful trajectories followed similar pathways.

To increase the samples of transitions to the ITC state, we performed 160 extra-simulations of $5 \times 10^{6} \mathrm{MD}$ steps in which the contact between DNA and the TFIIE Ewing was weakened intentionally (see Materials and Methods; Supplementary Figure S3). In this setup, we observed the successful transition to the ITC state for 102 out of 160 cases with the transition pathway unchanged. The rest of trajectories stayed at the intermediate state $I_{2}$ until the end of trajectories (58/160 cases).

In the ITC state, the DNA bubble size was, on average, $13.4 \pm$ 1.1 bp (Figure 4C; Supplementary Figure S3B), which perfectly agrees with the previous estimate (13. 4 bp) (Tomko et al., 2017).

\section{Fork Loop 1 Serve as a Gatekeeper}

The intermediate state $\mathrm{I}_{2}$ appears because of the blockage by the fork loop 1, which led us to hypothesize that the fork loop 1 may serve as a gatekeeper. To monitor large-scale motions of the fork loop 1, we plotted in Figure 4D the time courses of the distance between the fork loop 1 and the B-linker of TFIIB, finding that the fork loop 1 exhibits intermittent large-scale fluctuation to open the gate (green dashed lines in Figure 4D). In the representative time course (red), the time of the transition from $\mathrm{I}_{2}$ to ITC states in Figure 4B coincides with a large-scale opening. Looking into structure changes at the time, we found that the template DNA strand passed the fork loop 1 upon the loop opening, and moved toward the active site (Figure 4A; Supplementary FigureS4, right). Notably, in any trajectory, the non-template DNA strand never passed the fork loop 1. Instead, the non-template DNA strand approached to the wall of Pol II. Therefore, after the passage of the template strand, the fork loop 1 is located inside the DNA bubble. This support the hypothesis that the fork loop 1 serve as a gatekeeper; it is only passed by the template, but not the non-template DNA strand. This role is supported by previous studies (Plaschka et al., 2016; Meyer et al., 2006; Meyer et al., 2009).

The fork loop 1 sequence is fairly well conserved from yeast to human (Figure 5). In the human Pol II, it has been reported that a mutant that deletes two residues in the fork loop 1 (K458, A459 in human Pol II, which align with K471, A472 in yeast Pol II) abolishes the transcription in vitro (Jeronimo et al., 2004). This supports the crucial role of the fork loop 1. The mutation may 


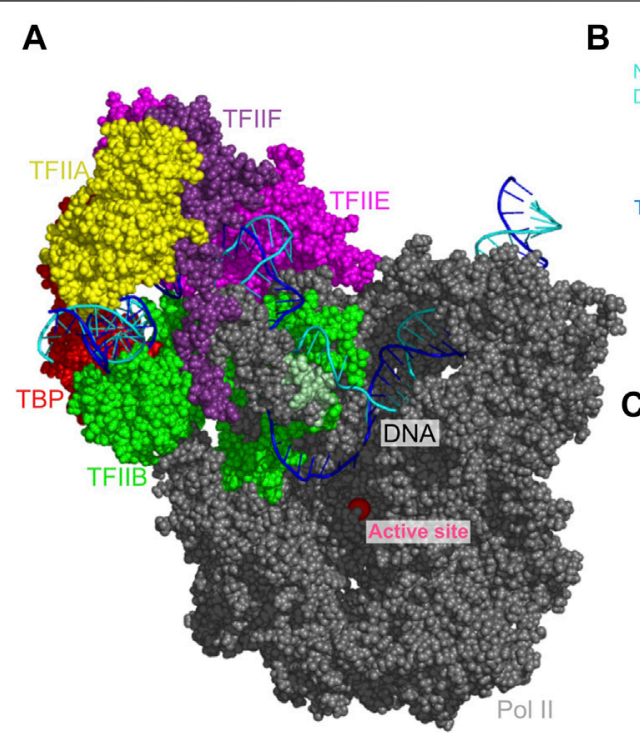

D

B

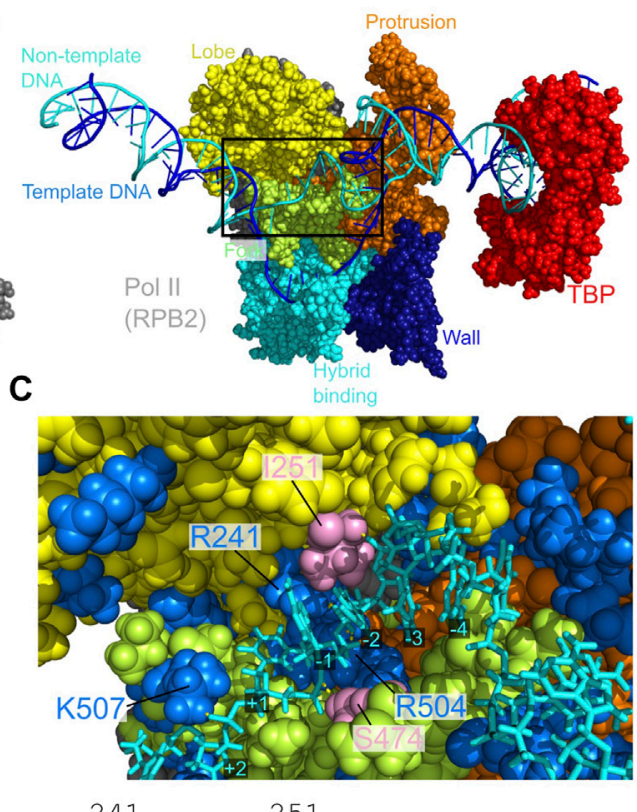

241
251

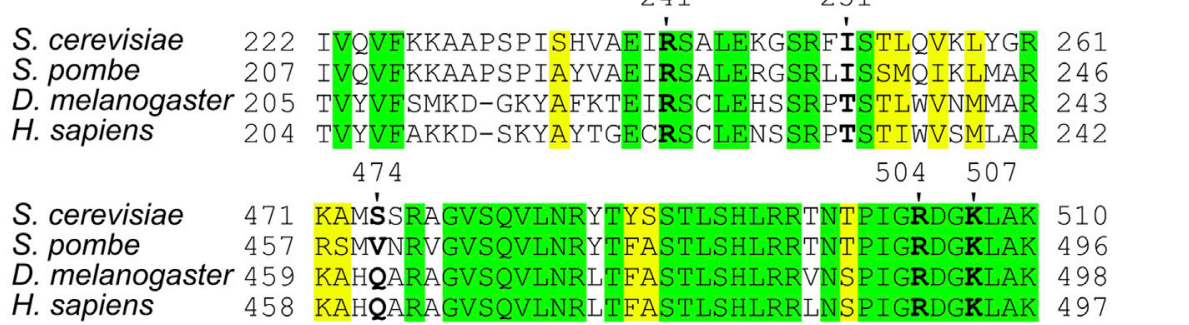

FIGURE 6 | The open DNA in the ITC state of PIC. (A) The atomic structure model for the ITC state. Some proteins are not displayed to make DNA visible. (B) The atomic structure model from the back side of (A), which focuses the non-template DNA strand path. (C) The close-up view of the Rpb2 and non-template DNA strand in the squared area in (B). Blue, positively charged residues; pink, I251 and S474 that form hydrogen bonds to DNA; yellow dashed lines, hydrogen bonds between bases of the non-template DNA strand and amino acids. (D) Multiple sequence alignment of the residues around those shown in (C). Green, invariant; yellow, conserved.

alter the loop opening dynamics, which led to the malfunction of Pol II.

\section{The Template and Non-Template DNA Strands in the Initially Transcribing Complex} To predict the placement of the template and non-template DNA strands and probe protein-DNA interactions in the ITC state, we constructed all-atom structure model via the back-mapping from snapshots in a CG-MD trajectory (Figure 6A), which is followed by $10 \mathrm{~ns}$ AA-MD simulations. We note that the template DNA strand was anticipated to be in the wall from the cryo-EM study even though the cryo-EM structure model for the ITC state does not contain the segment of the template and non-template DNA strands (Plaschka et al., 2016). The constructed model in this work supports this prediction; the template DNA strand is indeed placed in the wall of Pol II (Supplementary Figure S4, right). More specifically, our model suggests that the non-template DNA strand is localized at the protrusion, the lobe, and the fork of RPB2 subunit of Pol II (Figures 6B,C). This placement of the non-template DNA strand is fairly close to that found in the yeast elongation complex structures solved by X-ray diffraction (Supplementary Figure S5) (Barnes et al., 2015). These regions form the groove with many basic amino acids (Figure 6C). Along the groove the three basic residues, R241, R504, and K507 made specific interactions to the DNA at $-1,+1$, and +2 sites (Figure 6C). These three residues are strictly conserved across broad range of eukaryotes (Figure 6D).

\section{DISCUSSION}

Our computational modeling revealed that, after passing the OC state, the PIC passes two intermediate states before reaching the ITC, through which a small DNA bubble in the OC is expanded to complete the DNA bubble ready for RNA synthesis. One key gating state is $I_{2}$, where the upstream part of the template DNA strand ( -9 to -13 sites) interacts with the fork loop 1. The fluctuation of fork loop 1 was obligatory to escape from $\mathrm{I}_{2}$ that leads to engaging the template DNA into the active center at ITC (Figures $\mathbf{4}, \mathbf{5}$ ). The non-template DNA strand did not pass 
the fork loop 1, suggesting that the fork loop 1 serves as the gatekeeper for the DNA bubble.

Previous studies implicated two critical roles of fork loop 1. Based on the structural change in the fork loop 1 between the nucleic-acid free state and in the transcribing state (Meyer et al., 2006; Meyer et al., 2009) as well as the mutation assays (cite), the fork loop 1 was considered to play an important role in the transcription initiation. Alternatively, since the fork loop 1 is located at around the terminus of the DNA/RNA hybrid in the elongation complex, it may have important roles in separation of the product RNA from the template DNA strand. Our current simulation clearly supports the former functional role. The fork loop 1 forms a gate together with the rudder of RPB1 subunit in Pol II and serves as a gatekeeper for the engagement of the template DNA strand, but not the non-template DNA strand. The structural model obtained can be used to guess key residues as the gatekeeper, which can be examined by mutagenesis experiments. Furthermore, the shapes of the DNA transcription bubble of the intermediates $I_{1}$ and $I_{2}$ are different from those of OC and ITC. Especially, it will be interesting to investigate the structure change of the template strand (e.g., the opening of the DNA transcription bubble upstream) experimentally, for example, by the FRET technology.

Moreover, the current study found that non-template DNA strand in ITC is localized in the groove, formed by the protrusion, the lobe, and the fork of RPB2 subunit (Figure 6). This pass is close to the non-template DNA strand pass in the yeast elongation complex (Barnes et al., 2015), suggesting its ubiquitous importance. However, to our knowledge, these interaction sites were not investigated by mutagenesis. Systematic mutation assays in these sites would clarify the roles of stabilizing the non-template DNA strand in the transcription process.

In this study, we only mentioned the formation of DNA transcription bubble and did not discuss the initial transcription proceed by RNA polymerase. The ITC modeled in this study is a state in which transcription has not yet occurred, followed by the scanning of the transcription-start-site, early RNA transcription, and the promotor escape. It has been proposed that the initial transcription proceeds in prokaryotes via the "scrunching" model (Kapanidis et al., 2006; Winkelman and Gourse, 2017), but it is unclear whether the same is true for eukaryotic transcription.

Obviously missing in the current work is the kinetic and energetic arguments on the very initial process of the DNA opening in the transition from the $\mathrm{CC}$ to the $\mathrm{OC}$ states. In this study, even with the use of CG-MD simulations, the DNA opening was too slow to be simulated directly in MD simulations for the native promoter sequence. Instead, we needed to introduce a mismatch sequence in the promoter region. This is clearly a limitation. To study kinetic and energetic aspects in

\section{REFERENCES}

Abascal-Palacios, G., Ramsay, E. P., Beuron, F., Morris, E., and Vannini, A. (2018). Structural Basis of RNA Polymerase III Transcription Initiation. Nature 553, 301-306. doi:10.1038/nature25441 this initial DNA opening without the mismatch sequence, we need some advanced sampling methods, such as the umbrella sampling, the Markov-state modeling (Husic and Pande, 2018), and the string method (Weinan et al., 2002). Alternatively, since the ATP-driven motor activity of TFIIH helicase is expected to accelerate the DNA opening, including this effect either explicitly or implicitly may enable to simulate the dynamic process of the bubble formation more directly. These developments are left for future studies.

Related to this, it has been known that Pol I and Pol III systems do not have TFIIH-like helicases (Vannini and Cramer, 2012; Paule and White, 2000; Han et al., 2017; Gouge et al., 2017), yet initiating the transcription efficiently via similar three states (Engel et al., 2017; Abascal-Palacios et al., 2018; Vorlander et al., 2018). Comparison of the transcription initiation in the three RNA polymerase systems can put forward comprehensive understanding of transcription initiation in Eukaryote.

\section{DATA AVAILABILITY STATEMENT}

The original contributions presented in the study are included in the article/Supplementary Material, further inquiries can be directed to the corresponding author.

\section{AUTHOR CONTRIBUTIONS}

GS and ST contributed to conception and design of the study. GS performed simulations and analyzed data. GS and ST wrote the draft of the manuscript, contributed to manuscript revision, read, and approved the submitted version.

\section{FUNDING}

This work was supported by the Ministry of Education, Culture, Sport, Science, and Technology (MEXT) grant JPMXP1020200101 as "Program for Promoting Researches on the Supercomputer Fugaku" (ST), by the Japan Science and Technology Agency (JST) grant (JPMJCR1762) (ST), and by Japan Society for the Promotion of Science (Award number(s): 20H05934, 21H02441).

\section{SUPPLEMENTARY MATERIAL}

The Supplementary Material for this article can be found online at: https://www.frontiersin.org/articles/10.3389/fmolb.2021.772486/ full\#supplementary-material

Abraham, M. J., Murtola, T., Schulz, R., Páll, S., Smith, J. C., and Hess, B. (2015). Gromacs: High Performance Molecular Simulations through Multi-level Parallelism from Laptops to Supercomputers. SoftwareX 1-2, 19-25. doi:10.1016/j.softx.2015.06.001

Alekseev, S., Nagy, Z., Sandoz, J., Weiss, A., Egly, J.-M., Le May, N., et al. (2017). Transcription without XPB Establishes a Unified Helicase-independent 
Mechanism of Promoter Opening in Eukaryotic Gene Expression. Mol. Cel 65, 504-514. doi:10.1016/j.molcel.2017.01.012

Barnes, C. O., Calero, M., Malik, I., Graham, B. W., Spahr, H., Lin, G., et al. (2015). Crystal Structure of a Transcribing RNA Polymerase II Complex Reveals a Complete Transcription Bubble. Mol. Cel. 59, 258-269. doi:10.1016/ j.molcel.2015.06.034

Brandani, G. B., Niina, T., Tan, C., and Takada, S. (2018). DNA Sliding in Nucleosomes via Twist Defect Propagation Revealed by Molecular Simulations. Nucleic Acids Reserach 46 (6), 2788-2801. doi:10.1093/nar/gky158

Buratowski, S., Hahn, S., Guarente, L., and Sharp, P. A. (1989). Five Intermediate Complexes in Transcription Initiation by RNA Polymerase II. Cell 56 (4), 549-561. doi:10.1016/0092-8674(89)90578-3

Chen, J., Darst, S. A., and Thirumalai, D. (2010). Promoter Melting Triggered by Bacterial RNA Polymerase Occurs in Three Steps. Proc. Natl. Acad. Sci. USA 107 (28), 12523-12528. doi:10.1073/pnas.1003533107

Compe, E., and Egly, J.-M. (2012). TFIIH: when Transcription Met DNA Repair. Nat. Rev. Mol. Cel Biol 13 (6), 343-354. doi:10.1038/nrm3350

Dienemann, C., Schwalb, B., Schilbach, S., and Cramer, P. (2018). Promoter Distortion and Opening in the RNA Polymerase II Cleft. Mol. Cel 73, 97-e4. doi:10.1016/j.molcel.2018.10.014

Engel, C., Gubbey, T., Neyer, S., Sainsbury, S., Oberthuer, C., Baejen, C., et al. (2017). Structural Basis of RNA Polymerase I Transcription Initiation. Cell 169 (1), 120-131. doi:10.1016/j.cell.2017.03.003

Fazal, F. M., Meng, C. A., Murakami, K., Kornberg, R. D., and Block, S. M. (2015). Real-time Observation of the Initiation of RNA Polymerase II Transcription. Nature 525 (10), 274-277. doi:10.1038/nature14882

Feig, M., and Burton, Z. F. (2010). RNA Polymerase II with Open and Closed Trigger Loops: Active Site Dynamics and Nucleic Acid Translocation. Biophys. J. 9 (8), 577-2586. doi:10.1016/j.bpj.2010.08.010

Fiser, A., Do, R. K. G., and Šali, A. (2000). Modeling of Loops in Protein Structures. Protein Sci. 9, 1753-1773. doi:10.1110/ps.9.9.1753

Fishburn, J., Tomko, E., Galburt, E., and Hahn, S. (2015). Double-stranded DNA Translocase Activity of Transcription Factor TFIIH and the Mechanism of RNA Polymerase II Open Complex Formation. Proc. Natl. Acad. Sci. USA 112 (13), 3961-3966. doi:10.1073/pnas.1417709112

Forget, D., Langelier, M.-F., The'rien, C., Trinh, V., and Coulombe, B. (2004). Photo-Cross-Linking of a Purified Preinitiation Complex Reveals Central Roles for the RNA Polymerase II Mobile Clamp and TFIIE in Initiation Mechanisms. Mol. Cel Biol 24 (3), 1122-1131. doi:10.1128/mcb.24.3.1122-1131.2004

Freeman, G. S., Lequieu, J. P., Hinckley, D. M., Whitmer, J. K., and de Pablo, J. J. (2014). DNA Shape Dominates Sequence Affinity in Nucleosome Formation. Phys. Rev. Lett. 113, 168101. doi:10.1103/physrevlett.113.168101

Gouge, J., Guthertz, N., Kramm, K., Dergai, O., Abascal-Palacios, G., Satia, K., et al. (2017). Molecular Mechanisms of Bdp1 in TFIIIB Assembly and RNA Polymerase III Transcription Initiation. Nat. Commun. 8, 130. doi:10.1038/ s41467-017-00126-1

Grünberg, S., and Hahn, S. (2013). Structural Insights into Transcription Initiation by RNA Polymerase II. Trends Biochem. Sci. 38 (12), 603-611. doi:10.1016/ j.tibs.2013.09.002

Han, Y., Yan, C., Nguyen, T. H. D., Jackobel, A. J., Ivanov, I., Knutson, B. A., et al. (2017). Structural Mechanism of ATP-independent Transcription Initiation by RNA Polymerase I. eLife 6, e27414. doi:10.7554/eLife.27414

He, Y., Fang, J., Taatjes, D. J., and Nogales, E. (2013). Structural Visualization of Key Steps in Human Transcription Initiation. Nature 495, 481-486. doi:10.1038/nature11991

Hinckley, D. M., Freeman, G. S., Whitmer, J. K., and de Pablo, J. J. (2013). An Experimentally-Informed Coarse-Grained 3-Site-Per-Nucleotide Model of DNA: Structure, Thermodynamics, and Dynamics of Hybridization. J. Chem. Phys. 139, 144903. doi:10.1063/1.4822042

Huang, X., Wang, D., Weiss, D. R., Bushnell, D. A., Kornberg, R. D., and Levitt, M. (2010). RNA Polymerase II Trigger Loop Residues Stabilize and Position the Incoming Nucleotide Triphosphate in Transcription. Proc. Natl. Acad. Sci. 107 (36), 15745-15750. doi:10.1073/pnas.1009898107

Husic, B. E., and Pande, V. S. (2018). Markov State Models: From an Art to a Science. J. Am. Chem. Soc. 140 (7), 2386-2396. doi:10.1021/jacs.7b12191

Hyeon, C., and Thirumalai, D. (2011). Capturing the Essence of Folding and Functions of Biomolecules Using Coarse-Grained Models. Nat. Commun. 2, 487. doi:10.1038/ncomms1481
Ivani, I., Dans, P. D., Noy, A., Pérez, A., Faustino, I., Hospital, A., et al. (2016). Parmbsc1: A Refined Force Field for DNA Simulations. Nat. Methods 13, 55-58. doi:10.1038/nmeth.3658

Jeronimo, C., Langelier, M.-F., Zeghouf, M., Cojocaru, M., Bergeron, D., Baali, D., et al. (2004). RPAP1, a Novel Human RNA Polymerase II-Associated Protein Affinity Purified with Recombinant Wild-type and Mutated Polymerase Subunits. Mol. Cel Biol 24 (16), 7043-7058. doi:10.1128/mcb.24.16.70437058.2004

Jorgensen, W. L., Chandrasekhar, J., Madura, J. D., Impey, R. W., and Klein, M. L. (1983). Comparison of Simple Potential Functions for Simulating Liquid Water. J. Chem. Phys. 79, 926-935. doi:10.1063/1.445869

Kapanidis, A. N., Margeat, E., Ho, S. O., Kortkhonjia, E., Weiss, S., and Ebright, R. H. (2006). Initial Transcription by RNA Polymerase Proceeds through a DNAScrunching Mechanism. Science 314 (5802), 1144-1147. doi:10.1126/ science.1131399

Kenzaki, H., Koga, N., Hori, N., Kanada, R., Li, W., Okazaki, K., et al. (2011). CafeMol: A Coarse-Grained Biomolecular Simulator for Simulating Proteins at Work. J. Chem. Theor. Comput. 7 (6), 1979-1989. doi:10.1021/ct2001045

Kmiecik, S., Gront, D., Kolinski, M., Wieteska, L., Dawid, A. E., and Kolinski, A. (2016). Coarse-Grained Protein Models and Their Applications. Chem. Rev. 116 (14), 7898-7936. doi:10.1021/acs.chemrev.6b00163

Krivov, G. G., Shapovalov, M. V., and Dunbrack, R. L., Jr. (2009). Improved Prediction of Protein Side-Chain Conformations with SCWRL4. Proteins 77 (4), 778-795. doi:10.1002/prot.22488

Lequieu, J., Schwartz, D. C., and de Pablo, J. J. (2017). In Silico evidence for Sequence-dependent Nucleosome Sliding. Proc. Natl. Acad. Sci. USA 114 (44), E9197-E9205. doi:10.1073/pnas.1705685114

Levy, Y., Onuchic, J. N., and Wolynes, P. G. (2007). Fly-Casting in Protein-DNA Binding: Frustration between Protein Folding and Electrostatics Facilitates Target Recognition. J. Am. Chem. Soc. 129 (4), 738-739. doi:10.1021/ ja065531n

Li, W., Wang, W., and Takada, S. (2014). Energy Landscape Views for Interplays Among Folding, Binding, and Allostery of Calmodulin Domains. Proc. Natl. Acad. Sci. U S A. 111 (9), 10550-10555. doi:10.1073/pnas.1402768111

Li, W., Terakawa, T., Wang, W., and Takada, S. (2012). Energy Landscape and Multiroute Folding of Topologically Complex Proteins Adenylate Kinase and 2ouf-Knot. Proc. Natl. Acad. Sci. 109 (44), 17789-17794. doi:10.1073/ pnas. 1201807109

Liwo, A., Baranowski, M., Czaplewski, C., Gołaś, E., He, Y., Jagieła, D., et al. (2014). A Unified Coarse-Grained Model of Biological Macromolecules Based on Mean-Field Multipole-Multipole Interactions. J. Mol. Model. 20, 2306. doi:10.1007/s00894-014-2306-5

Maier, J. A., Martinez, C., Kasavajhala, K., Wickstrom, L., Hauser, K. E., and Simmerling, C. (2015). ff14SB: Improving the Accuracy of Protein Side Chain and Backbone Parameters from ff99SB. J. Chem. Theor. Comput. 11, 3696-3713. doi:10.1021/acs.jctc.5b00255

Martí-Renom, M. A., Stuart, A. C., Fiser, A., Sánchez, R., Melo, F., and Šali, A. (2000). Comparative Protein Structure Modeling of Genes and Genomes. Annu. Rev. Biophys. Biomol. Struct. 29, 291-325. doi:10.1146/ annurev.biophys.29.1.291

Meyer, P. A., Ye, P., Suh, M.-H., Zhang, M., and Fu, J. (2009). Structure of the 12-Subunit RNA Polymerase II Refined with the Aid of Anomalous Diffraction Data. J. Biol. Chem. 284 (19), 12933-12939. doi:10.1074/ jbc.m809199200

Meyer, P. A., Ye, P., Zhang, M., Suh, M.-H., and Fu, J. (2006). Phasing RNA Polymerase II Using Intrinsically Bound Zn Atoms: An Updated Structural Model. Structure 14, 973-982. doi:10.1016/j.str.2006.04.003

Moore, B. L., Kelley, L. A., Barber, J., Murray, J. W., and MacDonald, J. T. (2013). High-quality Protein Backbone Reconstruction from Alpha Carbons Using Gaussian Mixture Models. J. Comput. Chem. 34 (22), 1881-1889. doi:10.1002/ jcc. 23330

Niina, T., Brandani, G. B., Tan, C., and Takada, S. (2017). Sequence-dependent Nucleosome Sliding in Rotation-Coupled and Uncoupled Modes Revealed by Molecular Simulations. Plos Comput. Biol. 13 (12), e1005880. doi:10.1371/ journal.pcbi.1005880

Nozawa, K., Schneider, T. R., and Cramer, P. (2017). Core Mediator Structure at 3.4 $\AA$ Extends Model of Transcription Initiation Complex. Nature 545, 248-251. doi:10.1038/nature22328 
Osman, S., and Cramer, P. (2020). Structural Biology of RNA Polymerase II Transcription: 20 Years on. Annu. Rev. Cel Dev. Biol. 36, 1-34. doi:10.1146/ annurev-cellbio-042020-021954

Pak, A. J., and Voth, G. A. (2018). Advances in Coarse-Grained Modeling of Macromolecular Complexes. Curr. Opin. Struct. Biol. 52, 119-126. doi:10.1016/ j.sbi.2018.11.005

Paule, M. R., and White, R. J. (2000). Survey and Summary Transcription by RNA Polymerases I and III. Nucleic Acids Res. 28 (6), 1283-1298. doi:10.1093/nar/ 28.6.1283

Plaschka, C., Hantsche, M., Dienemann, C., Burzinski, C., Plitzko, J., and Cramer, P. (2016). Transcription Initiation Complex Structures Elucidate DNA Opening. Nature 533, 353-358. doi:10.1038/nature17990

Plaschka, C., Larivière, L., Wenzeck, L., Seizl, M., Hemann, M., Tegunov, D., et al. (2015). Architecture of the RNA Polymerase II-Mediator Core Initiation Complex. Nature 518, 376-380. doi:10.1038/nature14229

Roeder, R. (1996). The Role of General Initiation Factors in Transcription by RNA Polymerase II. Trends Biochem. Sci. 21 (9), 327-335. doi:10.1016/s09680004(96)10050-5

Sainsbury, S., Bernecky, C., and Cramer, P. (2015). Structural Basis of Transcription Initiation by RNA Polymerase II. Nat. Rev. Mol. Cel Biol 16, 129-143. doi:10.1038/nrm3952

Sali, A., and Blundell, T. L. (1993). Comparative Protein Modelling by Satisfaction of Spatial Restraints. J. Mol. Biol. 234, 779-815. doi:10.1006/jmbi.1993.1626

Schilbach, S., Hantsche, M., Tegunov, D., Dienemann, C., Wigge, C., Urlaub, H., et al. (2017). Structures of Transcription Pre-initiation Complex with TFIIH and Mediator. Nature 551, 204-209. doi:10.1038/nature24282

Shimizu, M., Noguchi, Y., Sakiyama, Y., Kawakami, H., Katayama, T., and Takada, S. (2016). Near-atomic Structural Model for Bacterial DNA Replication Initiation Complex and its Functional Insights. Proc. Natl. Acad. Sci. USA 113 (50), E8021-E8030. doi:10.1073/pnas.1609649113

Shimizu, M., and Takada, S. (2018). Reconstruction of Atomistic Structures from Coarse-Grained Models for Protein-DNA Complexes. J. Chem. Theor. Comput. 14 (3), 1682-1694. doi:10.1021/acs.jctc.7b00954

Silva, D.-A., Weiss, D. R., Pardo Avila, F., Da, L.-T., Levitt, M., Wang, D., et al. (2014). Millisecond Dynamics of RNA Polymerase II Translocation at Atomic Resolution. Proc. Natl. Acad. Sci. 111 (21), 7665-7670. doi:10.1073/ pnas. 1315751111

Takada, S., Kanada, R., Tan, C., Terakawa, T., Li, W., and Kenzaki, H. (2015). Modeling Structural Dynamics of Biomolecular Complexes by Coarse-Grained Molecular Simulations. Acc. Chem. Res. 48 (12), 3026-3035. doi:10.1021/ acs.accounts. 5 b00338

Tan, C., and Takada, S. (2020). Nucleosome Allostery in pioneer Transcription Factor Binding. Proc. Natl. Acad. Sci. USA 117 (34), 20586-20596. doi:10.1073/ pnas. 2005500117

Terakawa, T., Kenzaki, H., and Takada, S. (2012). p53 Searches on DNA by Rotation-Uncoupled Sliding at C-Terminal Tails and Restricted Hopping of
Core Domains. J. Am. Chem. Soc. 134 (35), 14555-14562. doi:10.1021/ ja305369u

Terakawa, T., and Takada, S. (2014). RESPAC: Method to Determine Partial Charges in Coarse-Grained Protein Model and its Application to DNABinding Proteins. J. Chem. Theor. Comput. 10, 711-721. doi:10.1021/ ct4007162

Tomko, E. J., Fishburn, J., Hahn, S., and Galburt, E. A. (2017). TFIIH Generates a SixBase-Pair Open Complex during RNAP II Transcription Initiation and Start-Site Scanning. Nat. Struct. Mol. Biol. 24 (12), 1139-1145. doi:10.1038/nsmb.3500

Unarta, I. C., Cao, S., Kubo, S., Wang, W., Cheung, P. P. H., Gao, X., et al. (2021). Role of Bacterial RNA Polymerase Gate Opening Dynamics in DNA Loading and Antibiotics Inhibition Elucidated by Quasi-Markov State Model. Proc. Nat. Acad. Sci. U.S.A. 118, e2024324118. doi:10.1073/pnas.2024324118

Vannini, A., and Cramer, P. (2012). Conservation between the RNA Polymerase I, II, and III Transcription Initiation Machineries. Mol. Cel 45 (4), 439-446. doi:10.1016/j.molcel.2012.01.023

Vorländer, M. K., Khatter, H., Wetzel, R., Hagen, W. J. H., and Müller, C. W. (2018). Molecular Mechanism of Promoter Opening by RNA Polymerase III. Nature 553, 295-300. doi:10.1038/nature25440

Webb, B., and Sali, A. (2016). Comparative Protein Structure Modeling Using Modeller. Curr. Protoc. Bioinformatics 54, 5.6.1-5.6.37. doi:10.1002/cpbi.3

Weinan, E., Ren, W., and Vanden-Eijnden, E. (2002). String Method for the Study of Rare Events. Phys. Rev. B 66, 052301. doi:10.1103/physrevb.66.052301

Winkelman, J. T., and Gourse, R. L. (2017). Open Complex DNA Scrunching: A Key to Transcription Start Site Selection and Promoter Escape. Bioessays 39 (2), 1600193. doi:10.1002/bies.201600193

Zhang, B., Zheng, W., Papoian, G. A., and Wolynes, P. G. (2016). Exploring the Free Energy Landscape of Nucleosomes. J. Am. Chem. Soc. 138 (26), 8126-8133. doi:10.1021/jacs.6b02893

Conflict of Interest: The authors declare that the research was conducted in the absence of any commercial or financial relationships that could be construed as a potential conflict of interest.

Publisher's Note: All claims expressed in this article are solely those of the authors and do not necessarily represent those of their affiliated organizations, or those of the publisher, the editors and the reviewers. Any product that may be evaluated in this article, or claim that may be made by its manufacturer, is not guaranteed or endorsed by the publisher.

Copyright (C) 2021 Shino and Takada. This is an open-access article distributed under the terms of the Creative Commons Attribution License (CC BY). The use, distribution or reproduction in other forums is permitted, provided the original author(s) and the copyright owner(s) are credited and that the original publication in this journal is cited, in accordance with accepted academic practice. No use, distribution or reproduction is permitted which does not comply with these terms. 